\title{
Improved integrability and boundedness of solutions to some high-order variational problems
}

\author{
MyKhailo V. VOITOVYCH \\ (Presented by I. I. Skrypnik)
}

\begin{abstract}
In this article, we give a series of results on the improved integrability and boundedness of solutions to several high-order variational problems with strengthened coercivity. In particular, we consider the homogeneous Dirichlet problem on the minimum of integral functionals and study variational inequalities with unilateral and bilateral obstacles and also with integral and gradient constraints.
\end{abstract}

2010 MSC. 35A15, 35J35, 35J87, 49J40.

Key words and phrases. High-order integral functional, variational inequality, strengthened coercivity, Stampacchia's method, integrability, boundedness.

\section{Introduction}

Let $n, m \in \mathbb{N}$ and $p \in \mathbb{R}$ be numbers such that $m \geqslant 2, p>1$, and $n>m p$. Let $\Omega$ be a bounded open set of $\mathbb{R}^{n}$. The known counterexamples $[6,18]$ to the nineteenth Hilbert problem demonstrate that elliptic partial differential equations of the form

$$
\sum_{|\alpha| \leqslant m}(-1)^{|\alpha|} D^{\alpha} \mathcal{A}_{\alpha}\left(x, u, \ldots, D^{m} u\right)=0 \quad \text { in } \Omega
$$

can have unbounded generalized solutions when the coefficients $\mathcal{A}_{\alpha}$ are smooth functions satisfying the ordinary growth and coercivity conditions for the Sobolev space $W^{m, p}(\Omega)$. However, in this situation, it is possible to extract a subclass of equations of the form (1.1) whose all generalized solutions are bounded and Hölder continuous [21]. This class of equations is characterized by a strengthened coercivity condition under which the 
natural energy space is the space $W^{m, p}(\Omega) \cap W^{1, q}(\Omega)$ with $q \in(m p, n)$. In a typical case, this condition means that, for some constant $C>0$ and every $x \in \Omega$ and $\xi=\left\{\xi_{\alpha} \in \mathbb{R}:|\alpha| \leqslant m\right\}$, the following inequality holds:

$$
\sum_{1 \leqslant|\alpha| \leqslant m} \mathcal{A}_{\alpha}(x, \xi) \xi_{\alpha} \geqslant C\left\{\sum_{|\alpha|=1}\left|\xi_{\alpha}\right|^{q}+\sum_{|\alpha|=m}\left|\xi_{\alpha}\right|^{p}\right\} .
$$

In [21], Skrypnik has begun the study of the regularity of generalized solutions of variational high-order problems with strengthened coercivity. In particular, the boundedness and the Hölder continuity of solutions to variational inequalities with strengthened coercivity and sufficiently regular data were proved in $[3,12,14]$. Analogous results were obtained in $[2,4,5]$ for minimizers of second-order integral functionals.

We observe that the proof of the boundedness and the Hölder continuity of generalized solutions to the variational problems in $[2-5,12,14,21]$ uses a modification of Moser's method [19]. Using an analogue of Stampacchia's method [22,23], a weaker (exact in the scale of the Lebesgue spaces) condition on integrability of data was established in $[15,24]$ to guarantee the boundedness of generalized solutions of nonlinear highorder equations with strengthened coercivity. Moreover, a dependence of summability of generalized solutions to these equations on integrability of data was described in $[15,24]$. This dependence shows how, with an increase in the Lebesgue summability exponent of the right-hand side of the equation, the summability of its generalized solutions improves until they become bounded and Hölder continuous.

In the present article, we extend the results of $[15,24]$ to high-order integral functionals and variational inequalities with strengthened coercivity. Thus, we supplement and strengthen some results of $[2-5,12,14,21]$, since we establish properties of solutions of variational problems before they become bounded and Hölder continuous. Furthermore, here we study variational problems on subsets of $W_{0}^{m, p}(\Omega) \cap W_{0}^{1, q}(\Omega)$ that are invariant under the operation of $k$-level truncation by smooth cut-off functions $\left\{h_{k}\right\}_{k>0} \subset C^{m}(\mathbb{R})$. By their properties, the functions $h_{k}$ are similar to the standard truncation functions $T_{k}(s)=\max \{\min \{s, k\},-k\}$, $k>0, s \in \mathbb{R}$, and are their natural substitutes in high-order problems with strengthened coercivity (see $[15,24,25])$. The collection of sets that are invariant under the truncation operation by the functions $h_{k}$ is much wider than that defined by the constraints dictated by using of Moser's method in $[2-5,12,14]$. In particular, it covers the unilateral and bilateral obstacle problems. Their consideration here became possible due to the use of the method of $[15,24,25]$.

The further content of the present article is divided into four sections. 
In Section 2, we give necessary preliminaries and useful auxiliary assertions. The main results of the article are stated in Section 3 and proved in Section 4. In Section 5, we give some important examples where all the hypotheses are verified.

\section{Preliminaries and auxiliary assertions}

\subsection{Initial assumptions}

Let $m, n \in \mathbb{N}, m \geqslant 3$, and $n>2(m-1)$. These inequalities imply that $n(m-1)-2>0$ and

$$
\frac{2 n(m-2)}{n(m-1)-2}<\frac{2(m-1)}{m}<\frac{n}{m}, \quad 1<\frac{2 n(m-2)}{n(m-1)-2}<2 .
$$

Let $p \in \mathbb{R}$ be a number such that

$$
\frac{2 n(m-2)}{n(m-1)-2}<p<\frac{n}{m} .
$$

From $(2.1)$, it follows that $p(m-1)-2(m-2)>0$.

We set

$$
\bar{p}=\frac{2 p}{p(m-1)-2(m-2)} .
$$

By virtue of $(2.1)$, we have $\max (\bar{p}, m p)<n$.

Let $q \in \mathbb{R}$ be a number such that $\max (\bar{p}, m p)<q<n$.

If $p<2(m-1) / m$, then $\bar{p}>m p$; but if $p \geqslant 2(m-1) / m$, then $\bar{p} \leqslant m p$.

For more details on the above-made assumptions on the numbers $m$, $n, p$ and $q$, we refer to $[10,25]$.

\subsection{Functional spaces}

Let $\Omega$ be a bounded open set in $\mathbb{R}^{n}$. For every $r \geqslant 1,\|\cdot\|_{r}$ is the norm in the usual Lebesgue space $L^{r}(\Omega)$. By $W_{m, p}^{1, q}(\Omega)$ we denote the set $W^{1, q}(\Omega) \cap W^{m, p}(\Omega)$, where $W^{1, q}(\Omega)$ and $W^{m, p}(\Omega)$ are the classical Sobolev spaces (see, e.g., [16, Chapter II, $\S 2]$ ). The set $W_{m, p}^{1, q}(\Omega)$ is a Banach space with the norm

$$
\|u\|=\|u\|_{W^{1, q}(\Omega)}+\sum_{|\alpha|=m}\left\|D^{\alpha} u\right\|_{p} .
$$

By $W_{0}^{1, q}(\Omega)$ and $\stackrel{\circ}{W}_{m, p}^{1, q}(\Omega)$, we denote the closures of the set $C_{0}^{\infty}(\Omega)$ in $W^{1, q}(\Omega)$ and $W_{m, p}^{1, q}(\Omega)$, respectively. 
Remark 2.1. If the parameters $m, n, p$, and $q$ are such that $m=2$, $p>1$, and $n>q>2 p$, then all the other assumptions of Subsection 2.1 are not needed for the definitions of the spaces $W_{2, p}^{1, q}(\Omega)$ and $\stackrel{\circ}{W}_{2, p}^{1, q}(\Omega)$.

We set $q^{*}=n q /(n-q)$. As is known (see, for instance, [16, Chapter II, §2]),

$$
\begin{gathered}
W_{0}^{1, q}(\Omega) \subset L^{q^{*}}(\Omega), \\
\forall u \in W_{0}^{1, q}(\Omega), \quad\|u\|_{q^{*}} \leqslant c_{n, q}\left(\sum_{|\alpha|=1} \int_{\Omega}\left|D^{\alpha} u\right|^{q} d x\right)^{1 / q},
\end{gathered}
$$

where $c_{n, q}$ is a positive constant depending only on $n$ and $q$.

Next, let $\Lambda_{m}$ be the set of all $n$-dimensional multiindices $\alpha$ such that $1 \leqslant|\alpha| \leqslant m$. For every $\alpha \in \Lambda_{m}$, we set

$$
\frac{1}{q_{\alpha}}=\frac{|\alpha|-1}{p(m-1)}+\frac{m-|\alpha|}{q(m-1)} .
$$

The next result (see [10, Lemma 3.2]) is a consequence of the NirenbergGagliardo interpolation inequality [20, Lection II, inequality 2.2].

Lemma 2.2. Let $u \in \stackrel{\circ}{W}_{m, p}^{1, q}(\Omega)$. Then, for every $\beta \in \Lambda_{m}, 2 \leqslant|\beta| \leqslant$ $m-1$, there exists the weak derivative $D^{\beta} u \in L^{q_{\beta}}(\Omega)$ and the following inequality holds:

$$
\left\|D^{\beta} u\right\|_{q_{\beta}} \leqslant C\left(\sum_{|\alpha|=m}\left\|D^{\alpha} u\right\|_{p}\right)^{\frac{|\beta|-1}{m-1}}\left(\sum_{|\alpha|=1}\left\|D^{\alpha} u\right\|_{q}\right)^{\frac{m-|\beta|}{m-1}},
$$

where $C$ is a positive constant depending only on $n, m, p$, and $q$.

The following result is something like a chain rule for weak derivatives in $\stackrel{\circ}{W}_{m, p}^{1, q}(\Omega)$ (see [10, Lemma 3.5]).

Lemma 2.3. Let $h \in C^{m}(\mathbb{R}), h(0)=0$, and let the function $h$ and its derivatives $h^{(i)}, i=1, \ldots, m$, be bounded in $\mathbb{R}$. Let $u \in \stackrel{\circ}{W}_{m, p}^{1, q}(\Omega)$. Then $h(u) \in \stackrel{\circ}{W}_{m, p}^{1, q}(\Omega)$ and the following assertions hold:

(i) if $\alpha \in \Lambda_{m}$ and $|\alpha|=1$, then $D^{\alpha} h(u)=h^{\prime}(u) D^{\alpha} u$ a.e. in $\Omega$;

(ii) if $\alpha \in \Lambda_{m}$ and $2 \leqslant|\alpha| \leqslant m$, then

$$
\begin{aligned}
& \left|D^{\alpha} h(u)-h^{\prime}(u) D^{\alpha} u\right| \\
& \quad \leqslant c_{n, m}\left(\sum_{i=2}^{|\alpha|}\left|h^{(i)}(u)\right|\right) \sum_{1 \leqslant|\beta|<|\alpha|}\left|D^{\beta} u\right|^{|\alpha| /|\beta|} \text { a.e. in } \Omega,
\end{aligned}
$$

where the positive constant $c_{n, m}$ depends only on $m$ and $n$. 
In particular, if $u \in \stackrel{\circ}{W}_{2, p}^{1, q}(\Omega), \alpha \in \Lambda_{2}$, and $|\alpha|=2$, then

$$
\left|D^{\alpha} h(u)-h^{\prime}(u) D^{\alpha} u\right| \leqslant\left|h^{\prime \prime}(u)\right| \sum_{|\beta|=1}\left|D^{\beta} u\right|^{2} \quad \text { a.e. in } \Omega \text {. }
$$

\subsection{Truncation functions}

To state the main results, we also need the notion of smooth $k$ truncation functions.

Definition 2.4. Let $k>0$. A function $h_{k}: \mathbb{R} \rightarrow \mathbb{R}$ is called an $k$ truncation function if it has the following properties:

(i) $h_{k} \in C^{m}(\mathbb{R}), h_{k}$ and its derivatives $h_{k}^{(i)}, i=1, \ldots, m$, are bounded in $\mathbb{R}$;

(ii) $h_{k}(-s)=-h_{k}(s)$ for any $s \in \mathbb{R}$;

(iii) $h_{k}(s)=s$ if $|s| \leqslant k$ and $0 \leqslant h_{k}^{\prime}<1$ if $|s|>k$.

For every $k>0$, we denote by $\mathcal{T}_{k}$ the set of all $k$-truncation functions.

Specific $k$-truncation functions will be used in Section 4 to prove the main results of the paper. Such functions play a role similar to that of the standard truncation functions $T_{k}(s)=\max \{\min \{s, k\},-k\}$ in applying of Stampacchia's method for second-order elliptic equations and variational inequalities, and also for integral functionals of the form $\int_{\Omega} F(x, u, \nabla u) d x$ (see, e.g., $[1,8,11,22,23])$.

\subsection{Stampacchia type lemmas}

For every Lebesgue measurable set $E \subset \Omega$, we denote by meas $E$ the $n$-dimensional Lebesgue measure of $E$.

The following three lemmas (see [15, Section 2]) are similar to the Stampacchia's results (see for instance [23, Section 4]) on the boundedness and summability of a measurable function $u: \Omega \rightarrow \mathbb{R}$ whose distribution function

$$
\varphi(s)=\text { meas }\{|u| \geqslant s\}, s \geqslant 0,
$$

satisfies certain special relations between $\varphi(l)$ and $\varphi(k)$ for $l>k$.

Lemma 2.5. Let $u: \Omega \rightarrow \mathbb{R}$ be a measurable function, $C>0,0 \leqslant \tau_{1}<$ $\tau_{2}, \gamma>1, k_{0} \geqslant 0$, and let the following inequality holds for any $k$ and $l$ such that $k_{0}<k<l<2 k$ :

$$
\varphi(l) \leqslant \frac{C k^{\tau_{1}}}{(l-k)^{\tau_{2}}}[\varphi(k)]^{\gamma} .
$$


Also assume that $\vartheta>k_{0}$ and

$$
\vartheta^{\tau_{2}-\tau_{1}} \geqslant 2^{\tau_{1}+(2 \gamma-1) \tau_{2} /(\gamma-1)} C\left[\varphi\left(k_{0}\right)\right]^{\gamma-1} .
$$

Then $u \in L^{\infty}(\Omega)$ and $\|u\|_{\infty}<k_{0}+\vartheta$.

Lemma 2.6. Let $u: \Omega \rightarrow \mathbb{R}$ be a measurable function, and let $C>0$, $0 \leqslant \tau_{1}<\tau_{2}$, and $k_{0} \geqslant 0$. Suppose that, for any $k$ and $l$ such that $k_{0}<k<l<2 k$, the following inequality holds:

$$
\varphi(l) \leqslant \frac{C k^{\tau_{1}}}{(l-k)^{\tau_{2}}} \varphi(k) .
$$

Then there exist positive constants $b$ and $K$ depending only on $\tau_{1}, \tau_{2}, k_{0}$, $C$ such that $\exp \left(b|u|^{\left(\tau_{2}-\tau_{1}\right) / \tau_{2}}\right) \in L^{1}(\Omega)$ and

$$
\int_{\Omega} \exp \left(b|u|^{\left(\tau_{2}-\tau_{1}\right) / \tau_{2}}\right) d x \leqslant K \text { meas } \Omega .
$$

Lemma 2.7. Let $u: \Omega \rightarrow \mathbb{R}$ be a measurable function, $C>0, \tau>0$, $\gamma \in(0,1), k_{0} \geqslant 0$ and the following inequality holds for any $k>k_{0}$ :

$$
\varphi(2 k) \leqslant C k^{-\tau}[\varphi(k)]^{\gamma} .
$$

Then, for every $\lambda \in(0, \tau /(1-\gamma))$, we have $u \in L^{\lambda}(\Omega)$, and $\|u\|_{\lambda} \leqslant K^{\prime}$, where $K^{\prime}$ is a positive number depending only on $C, \tau, \gamma, \lambda, k_{0}$, and meas $\Omega$.

\section{Main results}

\subsection{Summability of minimizers of integral functionals}

We denote by $\mathbb{R}^{n, m}$ the space of all sets $\xi=\left\{\xi_{\alpha} \in \mathbb{R}: \alpha \in \Lambda_{m}\right\}$. For every $u \in \stackrel{\circ}{W}_{m, p}^{1, q}(\Omega)$, we define $\nabla_{m} u=\left\{D^{\alpha} u: \alpha \in \Lambda_{m}\right\}$.

We consider the functional $\mathcal{I}: \stackrel{\circ}{W}_{m, p}^{1, q}(\Omega) \rightarrow \mathbb{R}$ such that

$$
\forall u \in \stackrel{\circ}{W}_{m, p}^{1, q}(\Omega), \quad \mathcal{I}(u)=\int_{\Omega}\left\{F\left(x, \nabla_{m} u\right)+g(x, u)\right\} d x .
$$

We need the following structural hypotheses on $F$ and $g$ :

(H1) $F: \Omega \times \mathbb{R}^{n, m} \rightarrow \mathbb{R}$ and $g: \Omega \times \mathbb{R} \rightarrow \mathbb{R}$ are Carathéodory functions, i.e. for any $\xi \in \mathbb{R}^{n, m}$ and $s \in \mathbb{R}$, the functions $F(\cdot, \xi)$ and $g(\cdot, s)$ are measurable on $\Omega$ and for almost all $x \in \Omega$, the function $F(x, \cdot)$ is continuous in $\mathbb{R}^{n, m}$ and the function $g(x, \cdot)$ is continuous in $\mathbb{R}$. 
(H2) For almost all $x \in \Omega$, the function $F(x, \cdot)$ is convex in $\mathbb{R}^{n, m}$. This means that for almost all $x \in \Omega$ and every $\xi, \eta \in \mathbb{R}^{n, m}$ and $a \in[0,1]$,

$$
F(x, a \xi+(1-a) \eta) \leqslant a F(x, \xi)+(1-a) F(x, \eta) .
$$

(H3) Let $\left\{p_{\alpha}\right\}_{\alpha \in \Lambda_{m}}$ be a set of positive numbers such that

$$
\begin{gathered}
\frac{1}{p_{\alpha}}=\frac{|\alpha|-1}{p(m-1)}+\frac{m-|\alpha|}{q_{1}(m-1)} \quad \text { if } 2 \leqslant|\alpha| \leqslant m, \\
p_{\alpha}=q \quad \text { if }|\alpha|=1, \\
\max (\bar{p}, m p)<q_{1}<q<n .
\end{gathered}
$$

There exist $c_{1}, c_{2}, c_{3}>0$ and non-negative functions $f_{1}, f_{2} \in L^{1}(\Omega)$ such that, for almost all $x \in \Omega$ and every $\xi \in \mathbb{R}^{n, m}$, the following inequalities hold:

$$
\begin{gathered}
F(x, \xi) \geqslant c_{1} \sum_{|\alpha|=1, m}\left|\xi_{\alpha}\right|^{p_{\alpha}}-c_{2} \sum_{2 \leqslant|\alpha| \leqslant m-1}\left|\xi_{\alpha}\right|^{p_{\alpha}}-f_{1}(x), \\
F(x, \xi) \leqslant c_{3} \sum_{\alpha \in \Lambda_{m}}\left|\xi_{\alpha}\right|^{p_{\alpha}}+f_{2}(x) .
\end{gathered}
$$

(H4) There exist $c_{4}>0, \nu \in(0, q)$ and non-negative functions $g_{1}, g_{2} \in$ $L^{1}(\Omega)$ and $g_{3} \in L^{q^{*} /\left(q^{*}-\nu\right)}(\Omega)$ such that for almost all $x \in \Omega$ and every $s \in \mathbb{R}$ the following inequalities hold:

$$
\begin{gathered}
|g(x, s)| \leqslant c_{4}|s|^{q^{*}}+g_{1}(x), \\
g(x, s) \geqslant-|s|^{\nu} g_{3}(x)-g_{2}(x) .
\end{gathered}
$$

(H5) There exist $c_{5}>0, \mu \geqslant 1, k^{\prime}>0$ and a non-negative function $f_{3} \in L^{1}(\Omega)$ such that for almost all $x \in \Omega$ and every $k \geqslant k^{\prime}$ and $w, z \in \mathbb{R},|z| \geqslant|w| \geqslant k \geqslant k^{\prime}$ the following inequality holds:

$$
g(x, w)-g(x, z) \leqslant c_{5}(|w|+|z|)^{\mu-1}|w-z|+f_{3}(x)|w-z| .
$$

Remark 3.1. In view of relations (3.3)-(3.5), (3.6)-(3.8), inclusion (2.2), and Lemma 2.2, for every $u \in \stackrel{\circ}{W}_{m, p}^{1, q}(\Omega)$, the functions $F\left(x, \nabla_{m} u\right)$ and $g(x, u)$ are summable in $\Omega$ and the definition of functional (3.1) is correct. 
Remark 3.2. We note that due to (2.3), (2.4), (3.3)-(3.6), (3.9) and the Hölder's and Young's inequalities, functional (3.1) satisfies the following coercivity condition on $\stackrel{\circ}{W}_{m, p}^{1, q}(\Omega)$ (the so-called strengthened coercivity condition):

$$
\forall u \in \stackrel{\circ}{W}_{m, p}^{1, q}(\Omega), \quad \mathcal{I}(u) \geqslant C \int_{\Omega}\left\{\sum_{|\alpha|=1}\left|D^{\alpha} u\right|^{q}+\sum_{|\alpha|=m}\left|D^{\alpha} u\right|^{p}\right\} d x-C^{\prime},
$$

where $C, C^{\prime}$ are positive numbers depending only on $c_{1}, c_{2}, c_{n, q}, n, m$, $p, q, q_{1}, \nu,\left\|f_{1}\right\|_{1},\left\|g_{2}\right\|_{1},\left\|g_{3}\right\|_{q^{*} /\left(q^{*}-\nu\right)}$.

Remark 3.3. If, additionally to (3.2)-(3.9), for almost every $x \in \Omega$, the function $g(x, \cdot)$ is convex on $\mathbb{R}$ or the exponent $q^{*}$ in (3.8) is replaced by $\mu_{1} \in\left(0, q^{*}\right)$, and if $U$ is a nonempty sequentially weakly closed set in $\stackrel{\circ}{W}_{m, p}^{1, q}(\Omega)$, then there exists a minimizer $u \in U$ of the functional $\mathcal{I}$ on the set $U$ :

$$
\forall v \in U, \quad \mathcal{I}(u) \leqslant \mathcal{I}(v) .
$$

This fact follows from (3.11) and known results on the existence of minimum points of coercive functionals (see for instance [7, Section 6.2]). In addition, if $u$ is a minimizer of the functional $\mathcal{I}$ on $\stackrel{\circ}{W}_{m, p}^{1, q}(\Omega)$, then, by (3.11) and the inequalities

$$
\mathcal{I}(u) \leqslant \mathcal{I}(0) \leqslant \int_{\Omega}\left(g_{1}+f_{2}\right) d x,
$$

we have $\|u\| \leqslant C^{\prime \prime}$, where $C^{\prime \prime}$ is a positive number depending only on $c_{1}$, $c_{2}, c_{n, q}, n, m, p, q, q_{1}, \nu,\left\|f_{1}\right\|_{1},\left\|f_{2}\right\|_{1},\left\|g_{1}\right\|_{1},\left\|g_{2}\right\|_{1},\left\|g_{3}\right\|_{q^{*} /\left(q^{*}-\nu\right)}$.

Now, we are ready to give our main results. First, we state a theorem on the improved integrability and boundedness of minimizers of the functional $\mathcal{I}$.

Theorem 3.4. Let $r>q^{*} /\left(q^{*}-1\right)$. Suppose that hypotheses (H1)-(H5) hold with the functions $f_{1}, f_{2}$ and $f_{3}$ belonging to $L^{r}(\Omega)$, and let $M$ be a majorant for $\left\|f_{1}\right\|_{r},\left\|f_{2}\right\|_{r},\left\|f_{3}\right\|_{r},\left\|g_{1}\right\|_{1},\left\|g_{2}\right\|_{1}$ and $\left\|g_{3}\right\|_{q^{*} /\left(q^{*}-\nu\right)}$. Let $U$ be a nonempty set in $\stackrel{\circ}{W}_{m, p}^{1, q}(\Omega)$ satisfying the following condition:

there exists $k_{U} \geqslant 0$ such that if $k>k_{U}, h_{k} \in \mathcal{T}_{k}$ and $v \in U$, then $h_{k}(v) \in U$.

Finally, let $u \in U$ be a minimizer of the functional $\mathcal{I}$ on the set $U$. Then the following assertions hold:

(i) if $r<n / q, \mu \leqslant\left(q^{*}+r\right) / r$ and $q^{*}<\lambda<n r(q-1) /(n-q r)$, then $u \in L^{\lambda}(\Omega)$ and $\|u\|_{\lambda} \leqslant C_{1}$, where $C_{1}$ is a positive number depending 
only on $n, m, p, q, q_{1}, c_{1}, c_{2}, c_{3}, c_{5}, \nu, \mu, r, k^{\prime}, k_{U}, \lambda$, meas $\Omega$, $M$, and $\|u\|$;

(ii) if $r=n / q$ and $\mu \leqslant q^{*}-q+1$, then $\int_{\Omega} \exp \left(b|u|^{1 / \sigma}\right) d x \leqslant C_{2}$, where $\sigma>1$ depends only on $n, m, p, q, q_{1}$, and $b$ and $C_{2}$ are positive numbers depending only on $n, m, p, q, q_{1}, c_{1}, c_{2}, c_{3}, c_{5}, k^{\prime}, k_{U}, \nu$, $\mu$, meas $\Omega, M$, and $\|u\|$;

(iii) if $r>n / q$ and $\mu<q^{*}-q+1$, then $u \in L^{\infty}(\Omega)$ and $\|u\|_{\infty} \leqslant C_{3}$, where $C_{3}$ is a positive number depending only on $n, m, p, q, q_{1}$, $c_{1}, c_{2}, c_{3}, c_{5}, \nu, \mu, r, k^{\prime}, k_{U}$, meas $\Omega, M$, and $\|u\|$.

Remark 3.5. Let $U=\stackrel{\circ}{W}_{m, p}^{1, q}(\Omega)$. Then, by Lemma 2.3, condition (3.13) is satisfied with $k_{U}=0$. Furthermore, by virtue of Remark 3.3, the constants $C_{1}, C_{2}, C_{3}$ do not depend on $\|u\|$. In Section 5 , we give examples of non-empty closed convex subsets of $W_{m, p}^{1, q}(\Omega)$ which satisfy condition (3.13) and do not coincide with $\stackrel{\circ}{W}_{m, p}^{1, q}(\Omega)$. These are the sets that occur in the obstacle problems:

$$
\begin{gathered}
V_{\psi}=\left\{v \in \stackrel{\circ}{W}_{m, p}^{1, q}(\Omega): v \geqslant \psi \text { a.e. in } \Omega\right\} \quad \text { (a unilateral problem), } \\
V_{\psi_{1}, \psi_{2}}=\left\{v \in \stackrel{\circ}{W}_{m, p}^{1, q}(\Omega): \psi_{1} \leqslant v \leqslant \psi_{2} \text { a.e. in } \Omega\right\} \quad \text { (a bilateral problem), }
\end{gathered}
$$

as well as some other sets suggested by $[3,8,9,11-14]$ and defined by pointwise gradient and integral constraints. We also note that the form of condition (3.13) is similar to that of the corresponding conditions in some results of $[8,11]$, where the standard truncation functions $T_{k}$ were used instead of $h_{k}$.

Remark 3.6. To prove Theorem 3.4, we use a method close to that of $[15,24,25]$. This method is based on the use of Lemmas 2.5-2.7 and some family of $k$-truncation functions $\left\{h_{k}\right\}_{k>0}$ such that, for every $k>0$, $h_{k}^{\prime}(s)=0$ if $|s| \geqslant 2 k$ and $0<h_{k}^{\prime}(s)<1$ if $k<|s|<2 k$. The accurate definition of the functions $\left\{h_{k}\right\}_{k>0}$ will be given in the proof of Theorem 3.4. Condition (3.13) indicates that the set $U$ is closed (invariant) with respect to the operation of $k$-truncation by the functions $\left\{h_{k}\right\}_{k>0}$. This fact allows us to use $v=h_{k}(u)$ as test functions in (3.12) to obtain $\mathcal{I}(u) \leqslant \mathcal{I}\left(h_{k}(u)\right)$. From the last inequality, using the hypotheses (H2)(H5), the assumption $f_{1,2,3} \in L^{r}(\Omega)$ with $r>q^{*} /\left(q^{*}-1\right)$, as well as the structure of the functions $h_{k}(s)$ (especially on the set $k \leqslant|s| \leqslant 2 k$ ), we derive relations (2.6)-(2.8) with a constant $C>0$ independent of $u$ and a number $\gamma$ depending, in particular, on $r$. The subsequent application of Lemmas 2.5-2.7 establishes the validity of assertions (i)-(iii) of Theorem 3.4 . 
Remark 3.7. A result similar to Theorem 3.4 holds for minimizers of the second-order variational integral

$$
\int_{\Omega}\left\{F\left(x, \nabla_{2} u\right)+g(x, u)\right\} d x
$$

which is coercive on the space $\stackrel{\circ}{W}_{2, p}^{1, q}(\Omega)$. The proof of this fact is much simpler than the proof of Theorem 3.4, since there is no need to use Lemma 2.2 and some arguments (see [25]) related to the exponents $\left\{p_{\alpha}\right.$ : $2 \leqslant|\alpha| \leqslant m-1\}$ and the intermediate derivatives $\left\{D^{\alpha} u: 2 \leqslant|\alpha| \leqslant\right.$ $m-1\}$. In this case, instead of (2.1) and (3.5)-(3.7), we simply assume that $p>1, n>q>2 p$ and for almost all $x \in \Omega$ and every $\xi \in \mathbb{R}^{n, 2}$ the following inequalities hold:

$$
\begin{aligned}
c_{1}\left(\sum_{|\alpha|=1}\left|\xi_{\alpha}\right|^{q}+\sum_{|\alpha|=2}\left|\xi_{\alpha}\right|^{p}\right)-f_{1}(x) & \\
& \leqslant F(x, \xi) \leqslant c_{2}\left(\sum_{|\alpha|=1}\left|\xi_{\alpha}\right|^{q}+\sum_{|\alpha|=2}\left|\xi_{\alpha}\right|^{p}\right)+f_{2}(x) .
\end{aligned}
$$

\subsection{Summability of solutions to variational inequalities}

We now turn to the consideration of variational inequalities on subsets of the space $\stackrel{\circ}{W}_{m, p}^{1, q}(\Omega)$. We make the following hypotheses.

(H6) Let for every $\alpha \in \Lambda_{m}, A_{\alpha}: \Omega \times \mathbb{R}^{n, m} \rightarrow \mathbb{R}$ be a Carathéodory function, i.e. for any $\xi \in \mathbb{R}^{n, m}$, the function $A_{\alpha}(\cdot, \xi)$ is measurable on $\Omega$ and, for almost all $x \in \Omega$, the function $A_{\alpha}(x, \cdot)$ is continuous in $\mathbb{R}^{n, m}$.

(H7) There exist $c_{6}, c_{7}, c_{8}>0$ and non-negative functions $a_{1}, a_{2} \in L^{1}(\Omega)$ such that for almost all $x \in \Omega$ and every $\xi \in \mathbb{R}^{n, m}$ the following inequalities hold:

$$
\begin{gathered}
\sum_{|\alpha|=1, m} A_{\alpha}(x, \xi) \xi_{\alpha} \geqslant c_{6} \sum_{|\alpha|=1, m}\left|\xi_{\alpha}\right|^{p_{\alpha}}-c_{7} \sum_{2 \leqslant|\alpha| \leqslant m-1}\left|\xi_{\alpha}\right|^{p_{\alpha}}-a_{1}(x), \\
\sum_{\alpha \in \Lambda_{m}}\left|A_{\alpha}(x, \xi)\right|^{p_{\alpha} /\left(p_{\alpha}-1\right)} \leqslant c_{8} \sum_{\alpha \in \Lambda_{m}}\left|\xi_{\alpha}\right|^{p_{\alpha}}+a_{2}(x),
\end{gathered}
$$

where $\left\{p_{\alpha}\right\}_{\alpha \in \Lambda_{m}}$ is the set of positive numbers defined by means of $(3.3)-(3.5)$.

If the parameters $n, m, p$, and $q$ are such that $m=2, p>1$, $n>q>2 p$, then instead of (3.14) and (3.15), we assume that for 
almost all $x \in \Omega$ and any $\xi \in \mathbb{R}^{n, 2}$ the following inequalities hold:

$$
\begin{aligned}
\sum_{|\alpha|=1,2} A_{\alpha}(x, \xi) \xi_{\alpha} \geqslant & c_{6}\left\{\sum_{|\alpha|=1}\left|\xi_{\alpha}\right|^{q}+\sum_{|\alpha|=2}\left|\xi_{\alpha}\right|^{p}\right\}-a_{1}(x), \\
\sum_{|\alpha|=1}\left|A_{\alpha}(x, \xi)\right|^{q /(q-1)}+ & \sum_{|\alpha|=2}\left|A_{\alpha}(x, \xi)\right|^{p /(p-1)} \\
& \leqslant c_{8}\left\{\sum_{|\alpha|=1}\left|\xi_{\alpha}\right|^{q}+\sum_{|\alpha|=2}\left|\xi_{\alpha}\right|^{p}\right\}+a_{2}(x) .
\end{aligned}
$$

Let $\mathcal{A}: \stackrel{\circ}{W}_{m, p}^{1, q}(\Omega) \rightarrow\left(\stackrel{\circ}{W}_{m, p}^{1, q}(\Omega)\right)^{*}$ be the operator such that

$$
\forall u, w \in \stackrel{\circ}{W}_{m, p}^{1, q}(\Omega), \quad\langle\mathcal{A} u, w\rangle=\int_{\Omega}\left\{\sum_{\alpha \in \Lambda_{m}} A_{\alpha}\left(x, \nabla_{m} u\right) D^{\alpha} w\right\} d x .
$$

Observe that, by hypothesis (H6) and inequalities (2.4) and (3.15), for every $u, w \in \stackrel{\circ}{W}_{m, p}^{1, q}(\Omega)$ and every $\alpha \in \Lambda_{m}$, the function $A_{\alpha}\left(x, \nabla_{m} u\right) D^{\alpha} w$ is summable on $\Omega$. Hence, the definition of the operator $\mathcal{A}$ is correct.

Let $f \in L^{q^{*} /\left(q^{*}-1\right)}(\Omega)$, and let $U$ be a nonempty set in $\stackrel{\circ}{W}_{m, p}^{1, q}(\Omega)$.

We consider the following problem:

$$
\begin{gathered}
u \in U, \\
\forall v \in U, \quad\langle\mathcal{A} u, u-v\rangle \leqslant \int_{\Omega} f(u-v) d x .
\end{gathered}
$$

Remark 3.8. If, additionally to the hypotheses (H6) and (H7), the coefficients of the operator $\mathcal{A}$ satisfy a certain monotonicity condition and if the set $U$ is convex and closed in $\stackrel{\circ}{W}_{m, p}^{1, q}(\Omega)$, then there exists a solution to problem (3.16), (3.17). Furthermore, if the corresponding strong monotonicity condition holds (see [25, inequality 2.18]), and if the set $U$ is convex and closed in ${ }^{W}{ }_{m, p}^{1, q}(\Omega)$, then there exists a unique solution to problem (3.16), (3.17). These facts follow from the known results of the theory of monotone operators [17, Chapter 8].

We now state the second main result of the paper. This is a theorem on the improved integrability and boundedness of solutions to problem (3.16), (3.17).

Theorem 3.9. Let $f \in L^{r}(\Omega), r>q^{*} /\left(q^{*}-1\right)$. Suppose that the hypotheses (H6), (H7) hold with the functions $a_{1}, a_{2} \in L^{r}(\Omega)$, and let $M$ be a majorant for $\left\|a_{1}\right\|_{r},\left\|a_{2}\right\|_{r}$ and $\|f\|_{r}$. Suppose also that a nonempty set $U \subset \stackrel{\circ}{W}_{m, p}^{1, q}(\Omega)$ satisfies condition (3.13), and let $u$ be a solution to problem (3.16), (3.17). Then the following assertions hold: 
(i) if $r<n / q$ and $q^{*}<\lambda<n r(q-1) /(n-q r)$, then $u \in L^{\lambda}(\Omega)$ and $\|u\|_{\lambda} \leqslant C_{4}$, where $C_{4}$ is a positive number depending only on $n, m$, $p, q, q_{1}, c_{6}, c_{7}, c_{8}, r, \lambda$, meas $\Omega, M, k_{U}$, and $\|u\|$;

(ii) if $r=n / q$, then $\exp \left(b|u|^{1 / \sigma}\right) \in L^{1}(\Omega)$ and $\int_{\Omega} \exp \left(b|u|^{1 / \sigma}\right) d x \leqslant$ $C_{5}$, where $\sigma>1$ depends only on $n, m, p, q, q_{1}$, and $b$ and $C_{5}$ are positive numbers depending only on $n, m, p, q, q_{1}, c_{6}, c_{7}, c_{8}$, meas $\Omega, M, k_{U}$, and $\|u\|$;

(iii) if $r>n / q$, then $u \in L^{\infty}(\Omega)$ and $\|u\|_{\infty} \leqslant C_{6}$, where $C_{6}$ is a positive number depending only on $n, m, p, q, q_{1}, c_{6}, c_{7}, c_{8}, r$, meas $\Omega, M$, $k_{U}$, and $\|u\|$.

Remark 3.10. If $U=\stackrel{\circ}{W}_{m, p}^{1, q}(\Omega)$ and $u$ is a solution to problem (3.16), (3.17), then the constants $C_{4}, C_{5}, C_{6}$ in the statement of Theorem 3.9 do not depend on $k_{U}$ and $\|u\|$.

Remark 3.11. Let $r>n / q$ and all the other assumptions of Theorem 3.9 hold, let $u$ be a solution to problem (3.16), (3.17). Then, by assertion (iii) of Theorem 3.9, we have $u \in L^{\infty}(\Omega)$. This inclusion and [3, Theorem 1 ] imply that $u$ is locally Hölder continuous in $\Omega$ if the set $U$ satisfies the following hypothesis (see [3, Hypothesis 3]):

(H8) there exist $c>0$ and a non-negative function $a \in L^{r}(\Omega)$ such that, for every solution $u$ of problem (3.16), (3.17) and every $w \in$ $\stackrel{\circ}{W}_{m, p}^{1, q}(\Omega) \cap L^{\infty}(\Omega)$,

$$
\left|\langle\mathcal{A} u, w\rangle-\int_{\Omega} f w d x\right| \leqslant c \int_{\Omega} a|w| d x .
$$

In Section 5 (see Example 8), we consider a set $U \neq \stackrel{\circ}{W}_{m, p}^{1, q}(\Omega)$ that satisfies condition (3.13) and Hypothesis (H8) simultaneously. This set is suggested by [3, Example 2], [14, Corollary 4.2].

\section{Proofs of the main results}

\subsection{Proof of Theorem 3.4}

Let $r>q^{*} /\left(q^{*}-1\right)$, let the hypotheses (H1)-(H5) be satisfied with the functions

$$
f_{1}, f_{2}, f_{3} \in L^{r}(\Omega), \quad g_{1}, g_{2} \in L^{1}(\Omega), \quad g_{3} \in L^{q^{*} /\left(q^{*}-\nu\right)}(\Omega),
$$

and let $M$ be a majorant for $\left\|f_{1}\right\|_{r},\left\|f_{2}\right\|_{r},\left\|f_{3}\right\|_{r},\left\|g_{1}\right\|_{1},\left\|g_{2}\right\|_{1}$ and $\left\|g_{3}\right\|_{q^{*} /\left(q^{*}-\nu\right)}$. Suppose also that $\mu<q^{*}$. Let $U$ be a nonempty set 
in $\stackrel{\circ}{W}_{m, p}^{1, q}(\Omega)$ satisfying condition (3.13), and let $u \in U$ be a minimizer of the functional $\mathcal{I}$ on the set $U$. Finally, let $\varphi$ be the function on $[0,+\infty)$ defined by (2.5). Our main goal is to establish for this function relations of the form (2.6)-(2.8) with a number $\gamma$, depending, in particular, on $r$.

By $c_{i}, i=9,10, \ldots$, we denote positive numbers depending only on $n, m, p, q, q_{1}, c_{1}, c_{2}, c_{3}, c_{4}, \nu, \mu, r, k^{\prime}, k_{U},\|u\|$, meas $\Omega$, and $M$.

Step 1. We now introduce some auxiliary numbers. By virtue of the assumption $r>q^{*} /\left(q^{*}-1\right)$, we have $(r-1) / r-1 / q^{*}>0$. We set

$$
\begin{gathered}
r_{1}=\left(\frac{r-1}{r}-\frac{1}{q^{*}}\right)^{-1} \\
\gamma=\min \left\{\frac{q^{*}}{r_{1}(q-1)}, \frac{q^{*}(r-1)}{q r}, \frac{q^{*}-\mu}{q-1}\right\} .
\end{gathered}
$$

By (4.1), we have

$$
\frac{1}{r_{1}}+\frac{1}{r}+\frac{1}{q^{*}}=1
$$

It is obvious that

$$
\frac{1}{q^{*}}+\frac{\mu-1}{q^{*}}+\frac{q^{*}-\mu}{q^{*}}=1 .
$$

In addition, it follows from the definition of the numbers $r_{1}$ and $\gamma$ that

$$
\begin{gathered}
\gamma=\frac{1}{q-1}\left(\frac{r-1}{r} q^{*}-1\right)<1 \text { if } r<\frac{n}{q} \text { and } \mu \leqslant \frac{q^{*}+r}{r}, \\
\gamma=1 \text { if } r=\frac{n}{q} \text { and } \mu \leqslant q^{*}-q+1, \\
\gamma>1 \text { if } r>\frac{n}{q} \text { and } \mu<q^{*}-q+1 .
\end{gathered}
$$

Step 2. We fix $k \geqslant k_{0}$, where $k_{0}$ is a positive number which will be specified below. We define a $k$-truncation function $h_{k}$ such as in [25].

Let $\Psi_{0}$ be a function on $[0,1]$ such that, for every $s \in[0,1]$,

$$
\Psi_{0}(s)=\int_{0}^{s} \rho^{t}(1-\rho)^{t} d \rho,
$$

where $t=t\left(n, m, p, q, q_{1}, r\right)$ is a sufficiently large positive number, which will be refined below. We set $a=1 / \Psi_{0}(1)$, and let $\Psi$ be a function on $[0,1]$ such that, for every $s \in[0,1]$,

$$
\Psi(s)=s-a \int_{0}^{s} \Psi_{0}(\rho) d \rho .
$$


Next, let $h_{k}$ be a function on $\mathbb{R}$ such that

$$
h_{k}(s)= \begin{cases}s & \text { if }|s| \leqslant k, \\ {\left[\Psi\left(\frac{|s|-k}{k}\right)+1\right] k \operatorname{sign} s} & \text { if } k<|s|<2 k, \\ {[\Psi(1)+1] k \operatorname{sign} s} & \text { if }|s| \geqslant 2 k .\end{cases}
$$

We consider some properties of the function $h_{k}$ which are needed in this paper. By $K_{i}, i=1,2, \ldots$, we denote positive numbers depending only on $t$ and $m$. We have

$$
\begin{gathered}
h_{k} \in C^{m}(\mathbb{R}), \\
\left|h_{k}\right|<2 k \text { on } \mathbb{R}, \\
h_{k}^{\prime}(s)=1 \text { if }|s| \leqslant k, \\
0<h_{k}^{\prime}(s)<1 \text { if } k<|s|<2 k, \\
h_{k}^{\prime}(s)=0 \text { if }|s| \geqslant 2 k, \\
\left|h_{k}^{(i)}\right| \leqslant \frac{K_{1}}{k^{i-1}} \text { on } \mathbb{R}, \quad i=2,3, \ldots, m .
\end{gathered}
$$

Moreover, the following assertions hold:

if $\delta \in(0,1 / 2), s \in \mathbb{R},|s| \in(k,(1+\delta) k] \cup[(2-\delta) k, 2 k), \alpha \in \Lambda_{m}$, and $2 \leqslant|\alpha| \leqslant m$, then

$$
\frac{\left|h_{k}^{(i)}(s)\right|^{p_{\alpha}}}{\left(1-h_{k}^{\prime}(s)\right)^{p_{\alpha}-1}} \leqslant \frac{K_{2} \delta^{t-(i-1) p_{\alpha}}}{k^{(i-1) p_{\alpha}}}, \quad i=2,3, \ldots,|\alpha| ;
$$

if $\delta \in(0,1 / 2), s \in \mathbb{R}$, and $(1+\delta) k \leqslant|s| \leqslant(2-\delta) k$, then

$$
\left|h_{k}^{(i)}(s)\right| \leqslant \frac{K_{3}\left(1-h_{k}^{\prime}(s)\right)}{(k \delta)^{i-1}}, \quad i=2,3, \ldots, m ;
$$

if $k<l \leqslant 2 k, s \in \mathbb{R}$, and $|s| \geqslant l$, then

$$
\left|s-h_{k}(s)\right| \geqslant K_{4} k\left(\frac{l-k}{k}\right)^{t+2} .
$$

Proofs of assertions (4.15) and (4.16) are given in [25]. It remains to prove assertion (4.14). In fact, let $\alpha \in \Lambda_{m}, 2 \leqslant i \leqslant|\alpha| \leqslant m, \delta \in(0,1 / 2)$, $s \in \mathbb{R}$,

$$
|s| \in(k,(1+\delta) k] \cup[(2-\delta) k, 2 k) \text { and } y=(|s|-k) / k .
$$


We estimate $\left|h_{k}^{(i)}(s)\right|$ from above. By the definition of $h_{k}$ and the Leibniz formula, we have

$$
h_{k}^{(i)}(s)=-\frac{a(\operatorname{sign} s)^{i+1}}{k^{i-1}} \sum_{j=0}^{i-2} C_{i-2}^{j} w^{(j)}(y) \nu^{(i-2-j)}(y),
$$

where $w(y)=y^{t}, \nu(y)=(1-y)^{t}$ and, for every $0 \leqslant j \leqslant i-2$,

$$
\begin{gathered}
w^{(j)}(y)=t(t-1) \ldots(t-j+1) y^{t-j}, \\
\nu^{(i-2-j)}(y)=(-1)^{i-2-j} t(t-1) \ldots(t-i+j+3)(1-y)^{t-i+j+2} .
\end{gathered}
$$

The last two equalities and (4.17) yield

$$
\left|h_{k}^{(i)}(s)\right| \leqslant \frac{K_{5}}{k^{i-1}} \sum_{j=0}^{i-2} y^{t-j}(1-y)^{t-i+j+2} .
$$

Next, we estimate $1-h_{k}^{\prime}(s)$ from below. We use the definition of $h_{k}$ and integrate by parts to obtain

$$
\begin{aligned}
& 1-h_{k}^{\prime}(s)=a \int_{0}^{y} \rho^{t}(1-\rho)^{t} d \rho \\
& =\frac{a}{t+1} y^{t+1}(1-y)^{t}+\frac{a t}{t+1} \int_{0}^{y} \rho^{t+1}(1-\rho)^{t-1} d \rho \\
& \geqslant \frac{a}{t+1} y^{t+1}(1-y)^{t} .
\end{aligned}
$$

Now, if $k<|s| \leqslant(1+\delta) k$, then $0<y \leqslant \delta<\frac{1}{2}<1-\delta \leqslant 1-y<1$, and by (4.18) and (4.19), we have

$$
\frac{\left|h_{k}^{(i)}(s)\right|^{p_{\alpha}}}{\left(1-h_{k}^{\prime}(s)\right)^{p_{\alpha}-1}} \leqslant \frac{K_{2}}{k^{(i-1) p_{\alpha}}} \cdot \frac{y^{(t-i+2) p_{\alpha}}}{y^{(t+1)\left(p_{\alpha}-1\right)}} \leqslant \frac{K_{2} \delta^{t-(i-1) p_{\alpha}}}{k^{(i-1) p_{\alpha}}} .
$$

If $(2-\delta) k \leqslant|s|<2 k$, then $0<1-y \leqslant \delta<\frac{1}{2}<1-\delta \leqslant y<1$. Therefore, inequalities (4.18) and (4.19) yield

$$
\frac{\left|h_{k}^{(i)}(s)\right|^{p_{\alpha}}}{\left(1-h_{k}^{\prime}(s)\right)^{p_{\alpha}-1}} \leqslant \frac{K_{2}}{k^{(i-1) p_{\alpha}}} \cdot \frac{(1-y)^{(t-i+2) p_{\alpha}}}{(1-y)^{t\left(p_{\alpha}-1\right)}} \leqslant \frac{K_{2} \delta^{t-(i-1) p_{\alpha}}}{k^{(i-1) p_{\alpha}}} .
$$

Thus, assertion (4.14) is proved.

Step 3. Now, we proceed to the immediate derivation of relations (2.6)-(2.8). We can assume that $\varphi(k)>0$, otherwise inequalities $(2.6)-$ (2.8) hold. 
Assertion (4.16) implies that, for every $l \in(k, 2 k]$,

$$
\varphi(l) \leqslant \frac{k^{(t+1) q^{*}}}{K_{4}^{q^{*}}(l-k)^{(t+2) q^{*}}} \int_{\Omega}\left|u-h_{k}(u)\right|^{q^{*}} d x .
$$

By virtue of Lemma 2.3 and the properties (4.8)-(4.13), we have $h_{k}(u) \in$ $\stackrel{\circ}{W}_{m, p}^{1, q}(\Omega)$ and the following assertions hold:

if $\alpha \in \Lambda_{m}$ and $|\alpha|=1$, then

$$
D^{\alpha} h_{k}(u)=h_{k}^{\prime}(u) D^{\alpha} u \quad \text { a.e. in } \Omega ;
$$

if $\alpha \in \Lambda_{m}$ and $2 \leqslant|\alpha| \leqslant m$, then

$$
\begin{aligned}
& \left|D^{\alpha} h_{k}(u)-h_{k}^{\prime}(u) D^{\alpha} u\right| \\
& \quad \leqslant c_{n, m}\left(\sum_{i=2}^{|\alpha|}\left|h_{k}^{(i)}(u)\right|\right)\left(\sum_{1 \leqslant|\beta|<|\alpha|}\left|D^{\beta} u\right|^{|\alpha| /|\beta|}\right) \quad \text { a.e. in } \Omega .
\end{aligned}
$$

We set

$$
\Phi=\sum_{|\alpha|=1}\left|D^{\alpha} u\right|^{q}+\sum_{|\alpha|=m}\left|D^{\alpha} u\right|^{p} .
$$

By (2.3), (4.21) and (4.10)-(4.12), we have the inequality

$$
\left(\int_{\Omega}\left|u-h_{k}(u)\right|^{q^{*}} d x\right)^{1 / q^{*}} \leqslant c_{n, q}\left(\int_{\Omega} \Phi\left(1-h_{k}^{\prime}(u)\right) d x\right)^{1 / q}
$$

that together with (4.20) yields

$$
\varphi(l) \leqslant\left(\frac{c_{n, q}}{K_{4}}\right)^{q^{*}} \frac{k^{(t+1) q^{*}}}{(l-k)^{(t+2) q^{*}}}\left(\int_{\Omega} \Phi\left(1-h_{k}^{\prime}(u)\right) d x\right)^{q^{*} / q} .
$$

Our further arguments are aimed at obtaining the inequality

$$
\left(\int_{\Omega} \Phi\left(1-h_{k}^{\prime}(u)\right) d x\right)^{q^{*} / q} \leqslant c_{9}[\varphi(k)]^{\gamma} .
$$

After this, we will be able to apply Lemmas $2.5-2.7$ and complete the proof.

The definition of $h_{k}$ and the properties (4.8)-(4.13) ensure that $h_{k} \in$ $\mathcal{T}_{k}$. Consequently, by condition (3.13), we have $h_{k}(u) \in U$. This inclusion and (3.12) imply that $\mathcal{I}(u) \leqslant \mathcal{I}\left(h_{k}(u)\right)$. From this, using (3.1) and the fact that $h_{k}(u)=u$ and $\nabla_{m} h_{k}(u)=\nabla_{m} u$ almost everywhere on the set $\{|u| \leqslant k\}$, we obtain

$$
\int_{\{|u|>k\}} F\left(x, \nabla_{m} u\right) d x \leqslant \int_{\{|u|>k\}} F\left(x, \nabla_{m} h_{k}(u)\right) d x+I_{1},
$$


where

$$
I_{1}=\int_{\{|u|>k\}}\left(g\left(x, h_{k}(u)\right)-g(x, u)\right) d x .
$$

Using inequality (3.2) with

$$
a=h_{k}^{\prime}(u), \quad \xi=\nabla_{m} u, \quad \eta=\frac{\nabla_{m} h_{k}(u)-h_{k}^{\prime}(u) \nabla_{m} u}{1-h_{k}^{\prime}(u)},
$$

(4.11), (4.12), (3.7) and assertions (4.21) and (4.22), we establish

$$
\begin{aligned}
\int_{\{|u|>k\}} F\left(x, \nabla_{m} h_{k}(u)\right) d x & \\
& \leqslant \int_{\{|u|>k\}} h_{k}^{\prime}(u) F\left(x, \nabla_{m} u\right) d x+\int_{\{|u|>k\}} f_{2} d x+c_{10} I_{2},
\end{aligned}
$$

where

$$
I_{2}=\sum_{1 \leqslant|\beta|<|\alpha| \leqslant m} \sum_{i=2}^{|\alpha|} \int_{\{|u|>k\}} \frac{\left|h_{k}^{(i)}(u)\right|^{p_{\alpha}}}{\left(1-h_{k}^{\prime}(u)\right)^{p_{\alpha}-1}}\left|D^{\beta} u\right|^{p_{\alpha}|\alpha| /|\beta|} d x .
$$

From this and from (4.26), (3.6), (4.10)-(4.12), we deduce

$$
\begin{aligned}
c_{1} \int_{\{|u|>k\}} & \Phi\left(1-h_{k}^{\prime}(u)\right) d x \\
& \leqslant \int_{\{|u|>k\}}\left(f_{1}+f_{2}\right) d x+I_{1}+c_{10} I_{2}+c_{2} \sum_{2 \leqslant|\beta| \leqslant m-1} I_{\beta},
\end{aligned}
$$

where

$$
I_{\beta}=\int_{\Omega}\left|D^{\beta} u\right|^{p_{\beta}}\left(1-h_{k}^{\prime}(u)\right) d x, \quad \beta \in \Lambda_{m}, \quad 2 \leqslant|\beta| \leqslant m-1 .
$$

Let us obtain suitable estimates for the addends on the right-hand side of (4.28).

By Hölder's inequality, we have

$$
\int_{\{|u|>k\}}\left(f_{1}+f_{2}\right) d x \leqslant\left\|f_{1}+f_{2}\right\|_{r}[\varphi(k)]^{(r-1) / r} \leqslant 2 M[\varphi(k)]^{(r-1) / r} .
$$

Step 4 (estimate for $\left.I_{1}\right)$. By (3.10), we have

$$
I_{1} \leqslant c_{5} I_{1}^{\prime}+I_{1}^{\prime \prime},
$$


where

$$
\begin{gathered}
I_{1}^{\prime}=\int_{\{|u|>k\}}\left(\left|h_{k}(u)\right|+|u|\right)^{\mu-1}\left|u-h_{k}(u)\right| d x, \\
I_{1}^{\prime \prime}=\int_{\{|u|>k\}} f_{3}\left|u-h_{k}(u)\right| d x .
\end{gathered}
$$

Taking into account the facts that $h_{k}(s)=s$ for $s \in[-k, k]$ and $\left|h_{k}(s)\right| \leqslant$ $|s|$ for $s \in \mathbb{R}$ and using (2.3), (4.4), Hölder's inequality and (4.23), we obtain

$$
\begin{aligned}
I_{1}^{\prime} & \leqslant 2^{\mu-1} \int_{\{|u|>k\}}|u|^{\mu-1}\left|u-h_{k}(u)\right| d x \\
& \leqslant 2^{\mu-1}\left(\int_{\Omega}|u|^{q^{*}} d x\right)^{(\mu-1) / q^{*}}\left(\int_{\Omega}\left|u-h_{k}(u)\right|^{q^{*}} d x\right)^{1 / q^{*}}[\varphi(k)]^{\left(q^{*}-\mu\right) / q^{*}} \\
& \leqslant c_{11}\left(\int_{\Omega} \Phi\left(1-h_{k}^{\prime}(u)\right) d x\right)^{1 / q}[\varphi(k)]^{\left(q^{*}-\mu\right) / q^{*}} .
\end{aligned}
$$

From this and Young's inequality, it follows that

$$
I_{1}^{\prime} \leqslant \frac{c_{1}}{8 c_{5}} \int_{\Omega} \Phi\left(1-h_{k}^{\prime}(u)\right) d x+c_{12}[\varphi(k)]^{q\left(q^{*}-\mu\right) / q^{*}(q-1)} .
$$

We use the fact that $h_{k}(s)=s$ for $s \in[-k, k],(4.3)$, Hölder's inequality and (4.23) to obtain

$$
\begin{aligned}
& I_{1}^{\prime \prime} \leqslant[\varphi(k)]^{1 / r_{1}}\left\|f_{3}\right\|_{r}\left(\int_{\Omega}\left|u-h_{k}(u)\right|^{q^{*}} d x\right)^{1 / q^{*}} \\
& \leqslant c_{n, q} M[\varphi(k)]^{1 / r_{1}}\left(\int_{\Omega} \Phi\left(1-h_{k}^{\prime}(u)\right) d x\right)^{1 / q} .
\end{aligned}
$$

Hence, using Young's inequality, we deduce

$$
I_{1}^{\prime \prime} \leqslant \frac{c_{1}}{8} \int_{\Omega} \Phi\left(1-h_{k}^{\prime}(u)\right) d x+c_{13}[\varphi(k)]^{q /(q-1) r_{1}} .
$$

Now, using (4.31), (4.32) and (4.33), we obtain the estimate

$$
I_{1} \leqslant \frac{c_{1}}{4} \int_{\Omega} \Phi\left(1-h_{k}^{\prime}(u)\right) d x+c_{5} c_{12}[\varphi(k)]^{q\left(q^{*}-\mu\right) / q^{*}(q-1)}+c_{13}[\varphi(k)]^{q /(q-1) r_{1}} .
$$

Step 5. We proceed to estimate the terms $I_{2}$ and $\sum_{2 \leqslant|\beta| \leqslant m-1} I_{\beta}$ on the right-hand side of (4.28). This is the most essential point in the proof. In this case, a crucial role is played by assertions (4.14), (4.15) 
and a special choice of the parameters $t=t\left(n, m, p, q, q_{1}, r\right)$ and $k_{0}$ in the construction of the function $h_{k}$ on Step $\mathscr{2}$.

We set

$$
\begin{gathered}
d=\frac{(m-1)^{2} q_{1} p}{q_{1}-m p}, \quad \theta=\frac{2 q d}{q-q_{1}} \sum_{i=0}^{m-2}\left(\frac{n}{p}\right)^{i}, \quad t=1+(m-1)(d+\theta) r \\
q_{0}=\max \left\{\frac{|\beta| p_{\alpha} p_{\beta}}{|\beta| p_{\beta}-|\alpha| p_{\alpha}}: 1 \leqslant|\beta|<|\alpha| \leqslant m\right\} .
\end{gathered}
$$

We will need the following easily verified inequalities:

$$
\begin{aligned}
& |\beta| p_{\beta}>|\alpha| p_{\alpha} \quad \text { if } \quad 1 \leqslant|\beta|<|\alpha| \leqslant m, \\
& \frac{\theta(m-1)}{t-1}<\frac{1}{r} \quad \text { and } \quad 0<q_{0} \leqslant d<\theta .
\end{aligned}
$$

By virtue of (2.3), we have

$$
\varphi(s) \leqslant c_{14} s^{-q^{*}} \quad \text { for every } \quad s>0 .
$$

Hence, we can assume the number $k_{0} \geqslant 1$ so large that

$$
\varphi(s)<(1 / 2)^{t-1}<1 \quad \text { for every } s \geqslant k_{0} .
$$

We set

$$
\delta=[\varphi(k)]^{1 /(t-1)}, \quad \varepsilon=\delta^{m-1} .
$$

Since $k \geqslant k_{0}$, by virtue of $(4.38)$, we have $\delta \in(0,1 / 2)$ and $\varepsilon \in(0,1 / 2)$.

A suitable estimate for $\sum_{2 \leqslant|\beta| \leqslant m-1} I_{\beta}$ was obtained in [25, inequality $(3.31)]$ :

$$
\sum_{2 \leqslant|\beta| \leqslant m-1} I_{\beta} \leqslant \varepsilon \int_{\Omega} \Phi\left(1-h_{k}^{\prime}(u)\right) d x+c_{15}\left(\varepsilon^{-\theta} \varphi(k)+\varepsilon^{t}\right) .
$$

Now, we estimate $I_{2}$. For every $\alpha \in \Lambda_{m}$ with $|\alpha| \geqslant 2$ and every $i=2, \ldots,|\alpha|$, we denote by $H_{k, \alpha, i}$ a measurable function on the set $\{|u|>k\}$ such that

$$
H_{k, \alpha, i}=\frac{\left|h_{k}^{(i)}(u)\right|^{p_{\alpha}}}{\left(1-h_{k}^{\prime}(u)\right)^{p_{\alpha}-1}} \quad \text { a.e. on }\{|u|>k\}
$$

It is obvious that if $\alpha, \beta \in \Lambda_{m}$ and $1 \leqslant|\beta|<|\alpha| \leqslant m$, then

$$
\frac{|\alpha| p_{\alpha}}{|\beta| p_{\beta}}+\frac{|\beta| p_{\beta}-|\alpha| p_{\alpha}}{|\beta| p_{\beta}}=1
$$


Using this equality, (4.27), (4.41), (4.35) and the Young inequality, we obtain

$$
I_{2} \leqslant \sum_{1 \leqslant|\beta|<|\alpha| \leqslant m} \sum_{i=2}^{|\alpha|} \int_{\{|u|>k\}} \varepsilon^{p_{\beta}|\beta| /|\alpha|} H_{k, \alpha, i}^{p_{\beta}|\beta| / p_{\alpha}|\alpha|}\left|D^{\beta} u\right|^{p_{\beta}} d x+c_{16} \varphi(k) \varepsilon^{-q_{0}} .
$$

We fix $\alpha, \beta \in \Lambda_{m}, i \in \mathbb{N}$ such that $1 \leqslant|\beta|<|\alpha| \leqslant m$ and $2 \leqslant i \leqslant|\alpha|$. It is clear that

$$
\begin{aligned}
& \int_{\{|u|>k\}} H_{k, \alpha, i}^{p_{\beta}|\beta| /|\alpha| p_{\alpha}}\left|D^{\beta} u\right|^{p_{\beta}} d x \\
& \quad=\int_{E_{k, \delta}^{\prime}} H_{k, \alpha, i}^{p_{\beta}|\beta| /|\alpha| p_{\alpha}}\left|D^{\beta} u\right|^{p_{\beta}} d x+\int_{E_{k, \delta}^{\prime \prime}} H_{k, \alpha, i}^{p_{\beta}|\beta| /|\alpha| p_{\alpha}}\left|D^{\beta} u\right|^{p_{\beta}} d x
\end{aligned}
$$

where

$$
\begin{aligned}
& E_{k, \delta}^{\prime}=\{k<|u| \leqslant k(1+\delta)\} \cup\{(2-\delta) k \leqslant|u|<2 k\} \\
& E_{k, \delta}^{\prime \prime}=\{k(1+\delta)<|u|<(2-\delta) k\} .
\end{aligned}
$$

By (4.41) and the assertions (4.14) and (4.15), we have

$$
\begin{gathered}
H_{k, \alpha, i} \leqslant \frac{K_{2} \delta^{t-(i-1) p_{\alpha}}}{k^{(i-1) p_{\alpha}}} \text { a.e. on } E_{k, \delta}^{\prime}, \\
H_{k, \alpha, i} \leqslant \frac{K_{3}^{p_{\alpha}}\left(1-h_{k}^{\prime}(u)\right)}{(k \delta)^{(i-1) p_{\alpha}}} \quad \text { a.e. on } E_{k, \delta}^{\prime \prime} .
\end{gathered}
$$

We use (4.39), (4.44) and (2.4) to obtain

$$
\varepsilon^{p_{\beta}|\beta| /|\alpha|} \int_{E_{k, \delta}^{\prime}} H_{k, \alpha, i}^{p_{\beta}|\beta| /|\alpha| p_{\alpha}}\left|D^{\beta} u\right|^{p_{\beta}} d x \leqslant c_{17}[\varphi(k)]^{t /(t-1)} .
$$

By (4.45), (4.39), (4.11), we have

$$
\varepsilon^{p_{\beta}|\beta| /|\alpha|} \int_{E_{k, \delta}^{\prime \prime}} H_{k, \alpha, i}^{p_{\beta}|\beta| /|\alpha| p_{\alpha}}\left|D^{\beta} u\right|^{p_{\beta}} d x \leqslant \frac{c_{18}}{k} \int_{\Omega}\left(1-h_{k}^{\prime}(u)\right)\left|D^{\beta} u\right|^{p_{\beta}} d x .
$$

Collecting (4.42), (4.43), (4.46), (4.47) and taking into account (4.29), (4.39), we obtain

$$
I_{2} \leqslant \frac{c_{19}}{k} \int_{\Omega} \Phi\left(1-h_{k}^{\prime}(u)\right) d x+\frac{c_{19}}{k} \sum_{2 \leqslant|\beta| \leqslant m-1} I_{\beta}+c_{19}[\varphi(k)]^{1-q_{0}(m-1) /(t-1)} .
$$


The last inequality together with (4.36)-(4.40) yields the desired estimate:

$$
c_{10} I_{2}+c_{2} \sum_{2 \leqslant|\beta| \leqslant m-1} I_{\beta} \leqslant \frac{c_{20}}{k^{(m-1) /(t-1)}} \int_{\Omega} \Phi\left(1-h_{k}^{\prime}(u)\right) d x+c_{20}[\varphi(k)]^{(r-1) / r} .
$$

Step 6. At this step, using the obtained estimates for the terms on the right-hand side of (4.28), we derive inequalities of the form (2.6)-(2.8) and complete the proof via Lemmas 2.5-2.7.

Without loss of generality, we assume that

$$
\frac{c_{20}}{k^{(m-1) /(t-1)}}<\frac{c_{1}}{4} \text {. }
$$

Taking into account the last inequality, we deduce from (4.28), (4.30), (4.34) and (4.48) the following inequality:

$$
\begin{aligned}
\frac{c_{1}}{2} \int_{\Omega} \Phi\left(1-h_{k}^{\prime}(u)\right) d x & \leqslant\left(2 M+c_{20}\right)[\varphi(k)]^{(r-1) / r} \\
& +c_{5} c_{12}[\varphi(k)]^{q\left(q^{*}-\mu\right) / q^{*}(q-1)}+c_{13}[\varphi(k)]^{q /(q-1) r_{1}} .
\end{aligned}
$$

Hence, taking into account (4.2) and (4.38), we obtain (4.25). In turn, (4.24) and (4.25) imply the assertion:

$$
\varphi(l) \leqslant \frac{c_{21} k^{(t+1) q^{*}}}{(l-k)^{(t+2) q^{*}}}[\varphi(k)]^{\gamma} \quad \text { if } \quad k_{0} \leqslant k<l \leqslant 2 k .
$$

Using this assertion, and also (4.5) and Lemma 2.7, we establish that assertion (i) of the theorem is valid. By virtue of assertion (4.49), relation (4.6) and Lemma 2.6, assertion (ii) of the theorem is true. Finally, using assertions (4.49), (4.7) and Lemma 2.5, we conclude that assertion (iii) of the theorem is also true.

\subsection{Proof of Theorem 3.9}

Suppose that the set $U$ satisfies condition (3.13), $u$ is a solution of problem (3.16), (3.17) and all the other assumptions of Theorem 3.9 are satisfied. Let $k>k_{U}$. We define the functions $\varphi$ and $h_{k}(u)$ in the same way as in the proof of Theorem 3.4. Substituting $v=h_{k}(u)$ in (3.17), we obtain

$$
\int_{\Omega}\left\{\sum_{\alpha \in \Lambda_{m}} A_{\alpha}\left(x, \nabla_{m} u\right) D^{\alpha}\left(u-h_{k}(u)\right)\right\} d x \leqslant \int_{\Omega} f\left(u-h_{k}(u)\right) d x .
$$

Reasoning as in the proof of [25, Theorem 2.2], we deduce from the last inequality that assertion (4.49) is true. Now, we complete the proof in the same way as the proof of Theorem 3.4. 


\section{Examples}

Consider some examples where the hypotheses (H1)-(H8) and condition (3.13) are satisfied.

Example 1. Let $n, m \in \mathbb{N}$ and $p, q, q_{1} \in \mathbb{R}$ be numbers such that $m \geqslant 3, p \geqslant 2$, and $m p<q_{1}<q<n$. Let $\Omega$ be a bounded open set in $\mathbb{R}^{n}$. Let $\left\{p_{\alpha}\right\}_{\alpha \in \Lambda_{m}}$ be the set of numbers defined by means of (3.3), (3.4). We define the function $F: \Omega \times \mathbb{R}^{n, m} \rightarrow \mathbb{R}$, setting for every $(x, \xi) \in \Omega \times \mathbb{R}^{n, m}$,

$$
F(x, \xi)=\sum_{\alpha \in \Lambda_{m}}\left|\xi_{\alpha}\right|^{p_{\alpha}}
$$

This function satisfies hypotheses (H1)-(H3).

Next, let $f \in L^{q^{*}} /\left(q^{*}-1\right)(\Omega)$. For every $(x, s) \in \Omega \times \mathbb{R}$, we define

$$
g(x, s)=|s|^{q^{*}}+s f(x) .
$$

It is easy to verify that the given function $g: \Omega \times \mathbb{R} \rightarrow \mathbb{R}$ satisfies the hypotheses (H1), (H4) and (H5).

Thus, in this example, the functional $\mathcal{I}$ takes the form:

$$
\mathcal{I}(u)=\int_{\Omega}\left\{\sum_{\alpha \in \Lambda_{m}}\left|D^{\alpha} u\right|^{p_{\alpha}}\right\} d x+\int_{\Omega}|u|^{q^{*}} d x+\int_{\Omega} f u d x, \quad u \in \stackrel{\circ}{W}_{m, p}^{1, q}(\Omega) .
$$

Concerning an example of functions satisfying hypotheses (H6), (H7), we refer to [25, Section 5].

The following four examples of sets are inspired by $[8,11]$.

First, we consider sets of constraints corresponding to unilateral problems.

Example 2. Let $\psi: \Omega \rightarrow \mathbb{R}$ be a measurable function such that

$$
\psi^{+}=\max (\psi, 0) \in L^{\infty}(\Omega) .
$$

We define

$$
V_{\psi}=\left\{v \in \stackrel{\circ}{W}_{m, p}^{1, q}(\Omega): v \geqslant \psi \text { a.e. in } \Omega\right\}
$$

and suppose that $V_{\psi} \neq \emptyset$. It is easy to see that $V_{\psi}$ is a convex closed set in $\stackrel{\circ}{W}_{m, p}^{1, q}(\Omega)$. We set $U=V_{\psi}$.

Let us show that condition (3.13) is satisfied. In fact, let $k_{U}=\left\|\psi^{+}\right\|_{\infty}$, $k>k_{U}, h_{k} \in \mathcal{T}_{k}$ and $v \in U$. It is obvious that

$$
\begin{gathered}
v \in \stackrel{\circ}{W}_{m, p}^{1, q}(\Omega), \\
v \geqslant \psi \quad \text { a.e. in } \Omega, \quad k \geqslant \psi \quad \text { a.e. in } \Omega .
\end{gathered}
$$


By virtue of (5.1), Definition 2.4 and Lemma 2.3, we have

$$
h_{k}(v) \in \stackrel{\circ}{W}_{m, p}^{1, q}(\Omega) .
$$

Next, in view of (5.2), there exists a set $E \subset \Omega$ of measure zero such that for every $x \in \Omega \backslash E$ we have $v(x) \geqslant \psi(x)$ and $k \geqslant \psi(x)$. Then, fixing $x \in \Omega \backslash E$ and using Definition 2.4, we obtain:

if $|v(x)| \leqslant k$, then $h_{k}(v(x))=v(x) \geqslant \psi(x)$;

if $v(x)>k$, then $\psi(x) \leqslant k \leqslant h_{k}(v(x))$;

if $v(x)<-k$, then $\psi(x) \leqslant v(x) \leqslant h_{k}(v(x))$.

Therefore, $h_{k}(v) \geqslant \psi$ a.e. in $\Omega$. This and (5.3) allow us to conclude that $h_{k}(v) \in U$. Thus, condition (3.13) is satisfied.

Now, we consider sets of constraints corresponding to bilateral problems.

Example 3. Let $\psi_{1}, \psi_{2}: \Omega \rightarrow \mathbb{R}$ be measurable functions such that

$$
\begin{gathered}
\psi_{1} \leqslant \psi_{2} \quad \text { a.e. in } \Omega, \\
\psi_{1}^{+}=\max \left(\psi_{1}, 0\right) \in L^{\infty}(\Omega), \quad \psi_{2}^{-}=-\min \left(\psi_{2}, 0\right) \in L^{\infty}(\Omega) .
\end{gathered}
$$

We define

$$
V_{\psi_{1}, \psi_{2}}=\left\{v \in \stackrel{\circ}{W}_{m, p}^{1, q}(\Omega): \psi_{1} \leqslant v \leqslant \psi_{2} \text { a.e. in } \Omega\right\}
$$

and suppose that $V_{\psi_{1}, \psi_{2}} \neq \emptyset$. The set $V_{\psi_{1}, \psi_{2}}$ is convex and closed in $\stackrel{\circ}{W}_{m, p}^{1, q}(\Omega)$.

Let us show that the set $U=V_{\psi_{1}, \psi_{2}}$ satisfies condition (3.13) with $k_{U}=\max \left(\left\|\psi_{1}^{+}\right\|_{\infty},\left\|\psi_{2}^{-}\right\|_{\infty}\right)$. In fact, let $k>k_{U}, h_{k} \in \mathcal{T}_{k}$ and $v \in U$. It is clear that $v \in \stackrel{\circ}{W}_{m, p}^{1, q}(\Omega)$,

$$
\begin{gathered}
\psi_{1} \leqslant v \leqslant \psi_{2} \quad \text { a.e. in } \Omega \\
\psi_{1} \leqslant k \quad \text { a.e. in } \Omega, \quad \psi_{2} \geqslant-k \quad \text { a.e. in } \Omega .
\end{gathered}
$$

In view of (5.4) and (5.5), there exists a set $E \subset \Omega$ of measure zero such that for every $x \in \Omega \backslash E$ we have $\psi_{1}(x) \leqslant v(x) \leqslant \psi_{2}(x), \psi_{1}(x) \leqslant k$ and $\psi_{2}(x) \geqslant-k$. Then, fixing $x \in \Omega \backslash E$ and using Definition 2.4, we obtain:

if $|v(x)| \leqslant k$, then $h_{k}(v(x))=v(x) \in\left[\psi_{1}(x), \psi_{2}(x)\right]$;

if $v(x)>k$, then $\psi_{1}(x) \leqslant k<h_{k}(v(x))<v(x) \leqslant \psi_{2}(x)$;

if $v(x)<-k$, then $\psi_{1}(x) \leqslant v(x)<h_{k}(v(x))<-k \leqslant \psi_{2}(x)$.

Therefore, $\psi_{1} \leqslant h_{k}(v) \leqslant \psi_{2}$ a.e. in $\Omega$. This and (5.3) imply that $h_{k}(v) \in$ $U$. Thus, condition (3.13) is satisfied.

In the following example, we define the set $U$ by means of a pointwise constraint for the gradients of functions in $\stackrel{\circ}{W}_{m, p}^{1, q}(\Omega)$. 
Example 4. We denote by $K_{n}(0)$ the totality of all nonempty closed convex sets of $\mathbb{R}^{n}$ that contain the origin. Let $K: \Omega \rightarrow K_{n}(0)$. In particular, for every $x \in \Omega$, we can define the set $K(x)$ as in [11, Examples 3.27, 3.28], [13, Examples 4.4,4.5]. For example, for every $x \in \Omega$, we can set $K(x)=\left\{\zeta \in \mathbb{R}^{n}:|\zeta| \leqslant \phi(x)\right\}$, where $\phi: \Omega \rightarrow \mathbb{R}$ is a nonnegative measurable function. Next, for every $u \in \stackrel{\circ}{W}_{m, p}^{1, q}(\Omega)$, we set $\nabla u=\left\{D^{\alpha} u\right.$ : $|\alpha|=1\}$. We define

$$
V(K)=\left\{v \in \stackrel{\circ}{W}_{m, p}^{1, q}(\Omega): \nabla u(x) \in K(x) \text { for a.e } x \in \Omega\right\} .
$$

It is easy to see that the set $V(K)$ is not empty, convex and closed in $\stackrel{\circ}{W}_{m, p}^{1, q}(\Omega)$.

We set $U=V(K)$. Let us show that condition (3.13) is satisfied. In fact, let $k>0, h_{k} \in \mathcal{T}_{k}$ and $v \in U$. It is obvious that $v \in \stackrel{\circ}{W}_{m, p}^{1, q}(\Omega)$,

$$
\begin{gathered}
\nabla v(x), 0 \in K(x) \text { for a.e. } x \in \Omega, \\
K(x) \text { is a convex set for a.e. } x \in \Omega .
\end{gathered}
$$

By Lemma 2.3, embedding (5.3) is valid. Moreover, the following equality holds:

$$
\nabla h_{k}(v)=h_{k}^{\prime}(v) \nabla v+\left(1-h_{k}^{\prime}(v)\right) 0 \text { a.e. in } \Omega .
$$

These facts, assertions (5.6), (5.7) and Definition 2.4 allow us to conclude that $h_{k}(v) \in \stackrel{\circ}{W}_{m, p}^{1, q}(\Omega)$ and $\nabla h_{k}(v(x)) \in K(x)$ for almost every $x \in \Omega$. Therefore, $h_{k}(v) \in U$. Thus, condition (3.13) is satisfied.

Next, we give an example of an integral constraint for the gradients of functions in $\stackrel{\circ}{W}_{m, p}^{1, q}(\Omega)$.

Example 5. Let $\lambda \in[1, q]$. We define

$$
U=\left\{v \in \stackrel{\circ}{W}_{m, p}^{1, q}(\Omega): \int_{\Omega}|\nabla v|^{\lambda} d x \leqslant 1\right\} .
$$

It is easy to see that $U$ is a nonempty convex closed set in $\mathscr{W}_{m, p}^{1, q}(\Omega)$. In the case $\lambda=1$, this set is an analogue of the sets considered in [11, Example 3.36], [17, Example 8.3]. Taking $k>0, h_{k} \in \mathcal{T}_{k}, v \in U$ and using Lemma 2.3 and Definition 2.4, we obtain $h_{k}(v) \in \stackrel{\circ}{W}_{m, p}^{1, q}(\Omega)$ and

$$
\int_{\Omega}\left|\nabla h_{k}(v)\right|^{\lambda} d x=\int_{\Omega}\left(h_{k}^{\prime}(v)\right)^{\lambda}|\nabla v|^{\lambda} d x \leqslant \int_{\Omega}|\nabla v|^{\lambda} d x \leqslant 1 .
$$

Hence, $h_{k}(v) \in U$. Thus, condition (3.13) is satisfied with $k_{U}=0$.

The following two examples were considered in [12, Examples 2,4]. 
Example 6. Let $\lambda \in\left[1, q^{*}\right]$. We define

$$
U=\left\{v \in \stackrel{\circ}{W}_{m, p}^{1, q}(\Omega):\|v\|_{\lambda} \leqslant 1\right\} .
$$

It is easy to see that $U$ is a nonempty convex closed set in $W_{m, p}^{1, q}(\Omega)$ which satisfies (3.13) because for any $k>0, h_{k} \in \mathcal{T}_{k}$ and $v \in \stackrel{\circ}{W}_{m, p}^{1, q}(\Omega)$, we have $\left|h_{k}(v)\right| \leqslant|v|$ a.e. in $\Omega$ and $h_{k}(v) \in \stackrel{\stackrel{1}{W}}{m, q}(\Omega)$.

Example 7. Let $Q: \Omega \times \mathbb{R} \rightarrow \mathbb{R}$ be a function such that for almost all $x \in \Omega$ the function $Q(x, \cdot)$ is convex on $\mathbb{R}$ and $Q(x, 0) \leqslant 0$. In particular (see [9, Example 1], [13, Example 4.2]), for every $x \in \Omega$ and $s \in \mathbb{R}$, we can set

$$
Q(x, s)= \pm s-b(x), \quad Q(x, s)=\left(s-b_{1}(x)\right)\left(s-b_{2}(x)\right),
$$

where $b, b_{1}, b_{2}: \Omega \rightarrow \mathbb{R}$ are functions in $\Omega$ such that $b \geqslant 0, b_{1} \leqslant 0 \leqslant b_{2}$ a.e. in $\Omega$.

We define

$$
U=\left\{v \in \stackrel{\circ}{W}_{m, p}^{1, q}(\Omega): Q(x, v(x)) \leqslant 0 \text { for a.e. } x \in \Omega\right\} .
$$

It is clear that the set $U$ is not empty, convex and closed in $\mathscr{W}_{m, p}^{1, q}(\Omega)$. Let us show that condition (3.13) is satisfied. In fact, let $k>0, h_{k} \in \mathcal{T}_{k}$ and $v \in U$. There exists a set $E \subset \Omega$ such that meas $E=0$ and for every $x \in \Omega \backslash E$, we have $Q(x, v(x)) \leqslant 0$. Then, fixing $x \in \Omega \backslash E$ and using Definition 2.4, we obtain:

$$
\begin{aligned}
& \text { if }|v(x)| \leqslant k \text {, then } Q\left(x, h_{k}(v(x))\right)=Q(x, v(x)) \leqslant 0 ; \\
& \text { if }|v(x)|>k \text {, then } 0<h_{k}(v(x)) / v(x)<1 \text { and } \\
& \begin{aligned}
Q\left(x, h_{k}(v(x))\right) & =Q\left(x, \frac{h_{k}(v(x))}{v(x)} v(x)+\left(1-\frac{h_{k}(v(x))}{v(x)}\right) 0\right) \\
\leqslant & \frac{h_{k}(v(x))}{v(x)} Q(x, v(x))+\left(1-\frac{h_{k}(v(x))}{v(x)}\right) Q(x, 0) \leqslant 0 .
\end{aligned}
\end{aligned}
$$

Therefore, $Q\left(x, h_{k}(v(x))\right) \leqslant 0$ for a.e. $x \in \Omega$. This and (5.3) imply that $h_{k}(v) \in U$. Thus, condition (3.13) is satisfied with $k_{U}=0$.

Now, we give an example of the integral constraints suggested by $[3,14]$.

Example 8. Let $a \in L^{q^{*} /\left(q^{*}-1\right)}(\Omega)$ and $a \geqslant 0$ a.e. in $\Omega$. We define

$$
U=\left\{v \in \stackrel{\circ}{W_{m, p}^{1, q}}(\Omega): \int_{\Omega} a|v| d x \leqslant 1\right\} .
$$


It is easy to see that $U$ is a nonempty convex closed set in $\mathscr{W}_{m, p}^{1, q}(\Omega)$ and in general $U \neq \stackrel{\circ}{W}_{m, p}^{1, q}(\Omega)$. Taking $k>0, h_{k} \in \mathcal{T}_{k}, v \in U$, and using (5.3) and the fact that $\left|h_{k}(v)\right| \leqslant|v|$ a.e. in $\Omega$, we obtain $h_{k}(v) \in W_{m, p}^{1, q}(\Omega)$ and

$$
\int_{\Omega} a\left|h_{k}(v)\right| d x \leqslant \int_{\Omega} a|v| d x \leqslant 1 .
$$

Hence, $h_{k}(v) \in U$. Thus, condition (3.13) is satisfied with $k_{U}=0$.

Let us consider problem (3.16), (3.17) with the set $U$ defined by (5.8). Suppose that $r>n / q, f \in L^{r}(\Omega)$ and hypotheses (H6), (H7) are satisfied with the functions $a_{1}, a_{2} \in L^{r}(\Omega)$. Let $u$ be a solution of problem (3.16), (3.17) with the set $U$ defined by (5.8). Then by assertion (iii) of Theorem 3.9 , we have

$$
u \in L^{\infty}(\Omega) .
$$

If, additionally, $a \in L^{r}(\Omega)$, then the set (5.8) satisfies hypothesis (H8) (for details, see [3]). This fact, (5.9) and [3, Theorem 1] imply that $u$ is locally Hölder continuous in $\Omega$.

Finally, we give an example which shows that the inequality $r>n / q$ in assertion (iii) of Theorem 3.9 in general cannot be weakened for the validity of this assertion.

Example 9. Suppose that $n>4, m=p=2,4<q<n, R \leqslant 1 / e$ and

$$
\Omega=\left\{x \in \mathbb{R}^{n}:|x|<R\right\} .
$$

Let for every $x \in \Omega$ and $\alpha=\left(\alpha_{1}, \ldots, \alpha_{n}\right) \in \Lambda_{2}$ with $|\alpha|=2$,

$$
A_{\alpha}(x, \xi)= \begin{cases}\xi_{\alpha} & \text { if } \exists i \in \mathbb{N}, 1 \leqslant i \leqslant n: \alpha_{i}=2, \\ 2 \xi_{\alpha} & \text { if } \alpha_{i} \neq 2 \forall i=1, \ldots, n,\end{cases}
$$

and for every $\alpha=\left(\alpha_{1}, \ldots, \alpha_{n}\right) \in \Lambda_{2}$ with $|\alpha|=1$,

$$
A_{\alpha}(x, \xi)=\left(\sum_{|\beta|=1} \xi_{\beta}^{2}\right)^{(q-2) / 2} \xi_{\alpha} .
$$

Then the functions $\left\{A_{\alpha}\right\}_{\alpha \in \Lambda_{2}}$ defined by (5.10), (5.11) satisfy hypotheses (H6) and (H7) with $a_{1,2} \equiv 0, c_{7}=0$ and the positive constants $c_{6}, c_{8}$ depending only on $n$ and $q$.

Next, we fix a function $\eta \in C^{\infty}([0, R])$ such that $0 \leqslant \eta \leqslant 1$ in $[0, R]$, $\eta=1$ in $[0, R / 2]$ and $\eta=0$ in $[3 R / 4, R]$. Let $u, f: \Omega \rightarrow \mathbb{R}$ be the functions such that $u(0)=f(0)=0$ and for every $x \in \Omega \backslash\{0\}$,

$$
u(x)=\eta(|x|) \ln |\ln | x||,
$$




$$
f(x)=\Delta^{2} u(x)-\Delta_{q} u(x),
$$

where

$$
\Delta^{2} u(x)=\sum_{i, j=1}^{n} \frac{\partial^{4} u(x)}{\partial x_{i}^{2} \partial x_{j}^{2}}, \Delta_{q} u(x)=\sum_{i=1}^{n} \frac{\partial}{\partial x_{i}}\left\{\left[\sum_{j=1}^{n}\left(\frac{\partial u(x)}{\partial x_{j}}\right)^{2}\right]^{\frac{q-2}{2}} \frac{\partial u(x)}{\partial x_{i}}\right\} .
$$

It is obvious that $u \notin L^{\infty}(\Omega)$ and

$$
f \in C(\bar{\Omega} \backslash\{0\}) .
$$

Direct calculations show that for every $x \in \Omega$ with $0<|x|<R / 2$,

$$
\begin{aligned}
& f(x)=\frac{2(n-2)(n-4)}{|x|^{4}|\ln | x||}-\frac{n^{2}-10 n+20}{|x|^{4} \ln ^{2}|x|}+\frac{4(n-4)}{|x|^{4} \ln ^{3}|x|} \\
& \quad-\frac{6}{|x|^{4} \ln ^{4}|x|}+\frac{q-1}{\left.|x|^{q}|\ln | x\right|^{q}}+\frac{n-q}{\left.|x|^{q}|\ln | x\right|^{q-1}} .
\end{aligned}
$$

From (5.14), (5.15) we obtain that $f \in L^{n / q}(\Omega)$ and $f \notin L^{\lambda}(\Omega)$ for any $\lambda>n / q$.

The routine arguments relating to (5.12), (5.13) and using spherical coordinates and integration by parts show that the function $u$ defined by (5.12) is a solution of problem (3.16), (3.17) with the coefficients defined by $(5.10)$ and (5.11), the right-hand side $f$ defined by (5.13) and the set $U=\stackrel{\circ}{W}_{2,2}^{1, q}(\Omega)$. Thus, we have $f \in L^{n / q}(\Omega)$ and all the conditions of Theorem 3.9 are satisfied except for the inclusion $f \in L^{r}(\Omega)$ with some $r>n / q$. However, $u \notin L^{\infty}(\Omega)$. This justifies that the requirement in assertion (iii) of Theorem 3.9 on the right-hand side of variational inequality (3.17) in general is unimprovable in the scale of Lebesgue spaces.

\section{Acknowledgements}

This research was supported by the State Fund for Fundamental Research of Ukraine (Project No. 0117U006053). The author thanks the anonymous referees for a series of useful remarks that allowed to improve the presentation of this article.

\section{References}

[1] L. Boccardo, The summability of solutions to variational problems since Guido Stampacchia // Rev. R. Acad. Cienc. Exactas Fis. Nat. Ser. A Math. RACSAM 97 (2003), No. 3, 413-421.

[2] S. Bonafede, On the boundedness of minimizers of some integral functionals with degenerate anisotropic intergrands // Rocky Mountain J. Math. (to appear). 
[3] S. Bonafede, S. D'Asero, Hölder continuity of solutions for a class of nonlinear elliptic variational inequalities of high order // Nonlinear Anal., 44 (2001), 657667.

[4] S. Bonafede, V. Cataldo, S. D'Asero, Hölder continuity up to the boundary of minimizers for some integral functionals with degenerate integrands // J. Appl. Math., (2007), Article ID 31819 doi:10.1155/2007/31819, 14 pp.

[5] S. D'Asero, V. Cataldo, F. Nicolosi, Regularity of minimizers of some integral functionals with degenerate integrands // Nonlinear Anal., 68 (2008), No. 11, 3283-3293.

[6] E. De Giorgi, Un esempio di estremali discontinue per un problema variazionale di tipo ellittico // Boll. Un. Mat. Ital., (4), 1 (1968), 135-137.

[7] P. Drábek, J. Milota, Methods of nonlinear analysis: applications to differential equations, Birkhäuser, Basel-Boston-Berlin, 2007.

[8] Y. S. Gorban, A. A. Kovalevsky, On the boundedness of solutions of degenerate anisotropic elliptic variational inequalities // Results. Math., 65 (2014), 121-142.

[9] A. A. Kovalevskii, On the uniform boundedness of solutions to nonlinear elliptic vatiational inequalities in variable domains // Differential Equations, 30 (1994), No. 8, 1270-1273.

[10] A. A. Kovalevsky, Entropy solutions of Dirichlet problem for a class of nonlinear elliptic high-order equations with $L^{1}$-data // Nonlinear Bound. Value Probl., 12 (2002), 119-127.

[11] A. A. Kovalevsky, Integrability and boundedness of solutions to some anisotropic problems // J. Math. Anal. Appl., 432 (2015), 820-843.

[12] A. Kovalevsky, F. Nicolosi, Boundedness of solutions of variational inequalities with nonlinear degenerated elliptic operators of high order // Appl. Anal., 65 (1997), 225-249.

[13] A. Kovalevsky, F. Nicolosi, Boundedness of solutions of degenerate nonlinear elliptic variational inequalities // Nonlinear Anal., 35 (1999), 987-999.

[14] A. Kovalevsky, F. Nicolosi, On Hölder continuity of solutions of equations and variational inequalities with degenerate nonlinear elliptic high order operators // Atti del $2^{\circ}$ Simposio Internazionale "Problemi attuali dell'analisi e della fisica matematica" dedicato alla memoria del Prof. Gaetano Fichera, Taormina, 15-17 ottobre 1998, Aracne, 205-220.

[15] A. A. Kovalevskii, M. V. Voitovich, On increasing the integrability of generalized solutions of the Dirichlet problem for fourth-order nonlinear equations with strengthened ellipticity // Ukrainian Math. J., 58 (2006), No. 11, 1717-1733.

[16] O. A. Ladyzhenskaya, N. N. Uraltseva, Linear and quasilinear elliptic equations, Nauka, Moscow, 1973.

[17] J.-L. Lions, Quelques Méthodes de Résolution des Problèmes aux Limites Non Linéaires, Dunod, Gauthier-Villars, Paris, 1969.

[18] V. G. Maz'ya, Examples of nonregular solutions of quasilinear elliptic equations with analytic coefficients // Funct. Anal. Appl., 2 (1968), No. 3, 230-234.

[19] J. Moser, A new proof of De Giorgi's theorem concerning the regularity problem for elliptic differential equations // Comm. Pure Appl. Math., 13 (1960), 457468. 
[20] L. Nirenberg, On elliptic partial differential equations // Ann. Scuola Norm. Sup. Pisa Cl. Sci., 13 (1959), No. 2, 115-162.

[21] I. V. Skrypnik, Higher order quasilinear elliptic equations with continuous generalized solutions // Differential Equations, 14 (1978), No. 6, 786-795.

[22] G. Stampacchia, Régularisation des solutions de problèmes aux limites elliptiques à données discontinues // Proc. Internat. Sympos. Linear Spaces (Jerusalem, 1960), Pergamon, Oxford, 1961, 399-408.

[23] G. Stampacchia, Équations elliptiques du second ordre à coefficients discontinus // Séminaire Jean Leray, 3 (1963-1964) 1-77.

[24] M. V. Voitovich, Integrability properties of generalized solutions of the Dirichlet problem for higher-order nonlinear equations with strengthened ellipticity // Tr. Inst. Prikl. Mat. Mekh., 15 (2007), 3-14 [in Russian].

[25] M. V. Voitovich, On the existence of bounded generalized solutions of the Dirichlet problem for a class of nonlinear high-order elliptic equations // J. Math. Sci. (N. Y.), 210 (2015), No. 1, 86-113.

\section{CONTACT INFORMATION}

Mykhailo

V. Voitovych
Institute of Applied Mathematics

and Mechanics,

National Academy of Sciences of Ukraine,

Sloviansk, Ukraine

E-Mail: voitovichmv76@gmail.com 